\title{
An Android App for IoT Based Remote Health Monitoring System
}

\author{
Smruthi K.M*, Tejas R $^{*}$ \\ Department of ECE, R.V. College of Engineering, Autonomous Institution Affiliated to VTU, \\ Belagavi, Karnataka, India
}

DOI: https://doi.org/10.21467/proceedings.1.63

* Corresponding author email: mnviju74@gmail.com

\begin{abstract}
Internet of Thing(IoT) has gained a demanding impact in the recent years with variety of applications, projects and real time requirements. This impact has motivated to contribute a Io'T based health care system using various Io'T based devices. This paper presents a project idea implemented on this ground highlighting the applications of IoT. The main contribution of this paper is to present the project carried out using IoT with a main objective is to evaluate the patient condition by analyzing the various parameters. This paper also gives a overview of various devices used for implementing IoT based health monitoring system.
\end{abstract}

Index Terms- Internet of Things, Raspberry Pi, Web Server, Aurdino IDE, Python.

\section{INTRODUCTION}

One of the major problems faced by the human community in this digital world is concerned more about the health. At the same time health sector has gained a huge impact with the advancement of the technology. IoT is one of the technologies creating a huge impact in health sector. Remote healthcare monitoring allows people to continue to stay at home rather than in expensive healthcare facilities such as hospitals or nursing homes. Wearable device with sensor enabled will be helpful to monitor the patience health in real time condition and motion activities. The Raspberry Pi which is a cheap, flexible, fully customizable and programmable small computer board brings the advantages of a PC to the domain of sensor network.

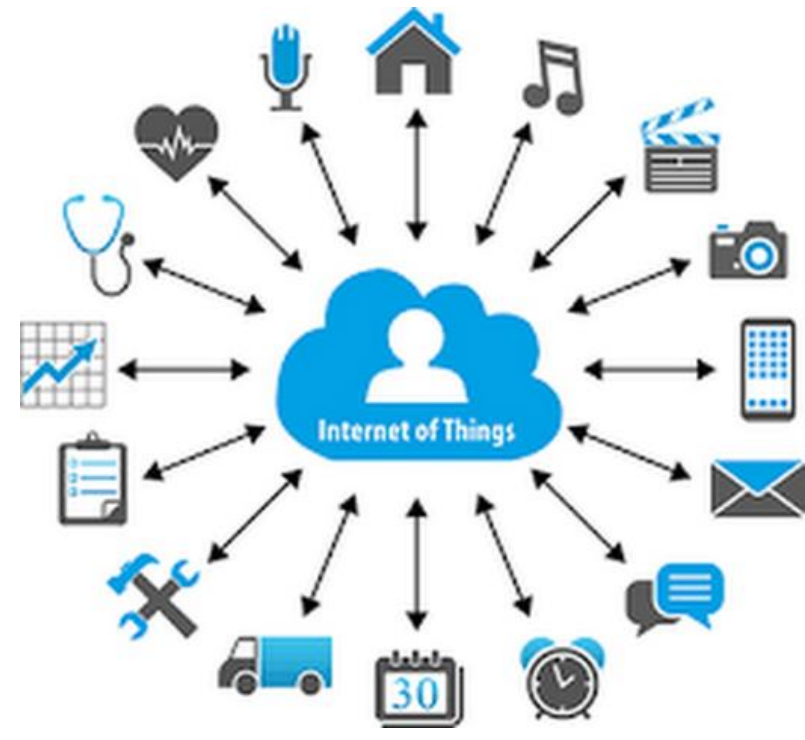

Figure-1: IoT Sectors

(C) 2018 Copyright held by the author(s). Published by AIJR Publisher in Proceedings of the $3^{\text {rd }}$ National Conference on Image Processing, Computing, Communication, Networking and Data Analytics (NCICCNDA 2018), April 28, 2018.

This is an open access article under Creative Commons Attribution-NonCommercial 4.0 International (CC BY-NC 4.0) ALjR license, which permits any non-commercial use, distribution, adaptation, and reproduction in any medium, as long as the
original work is properly cited. ISBN: 978-81-936820-0-5
} 


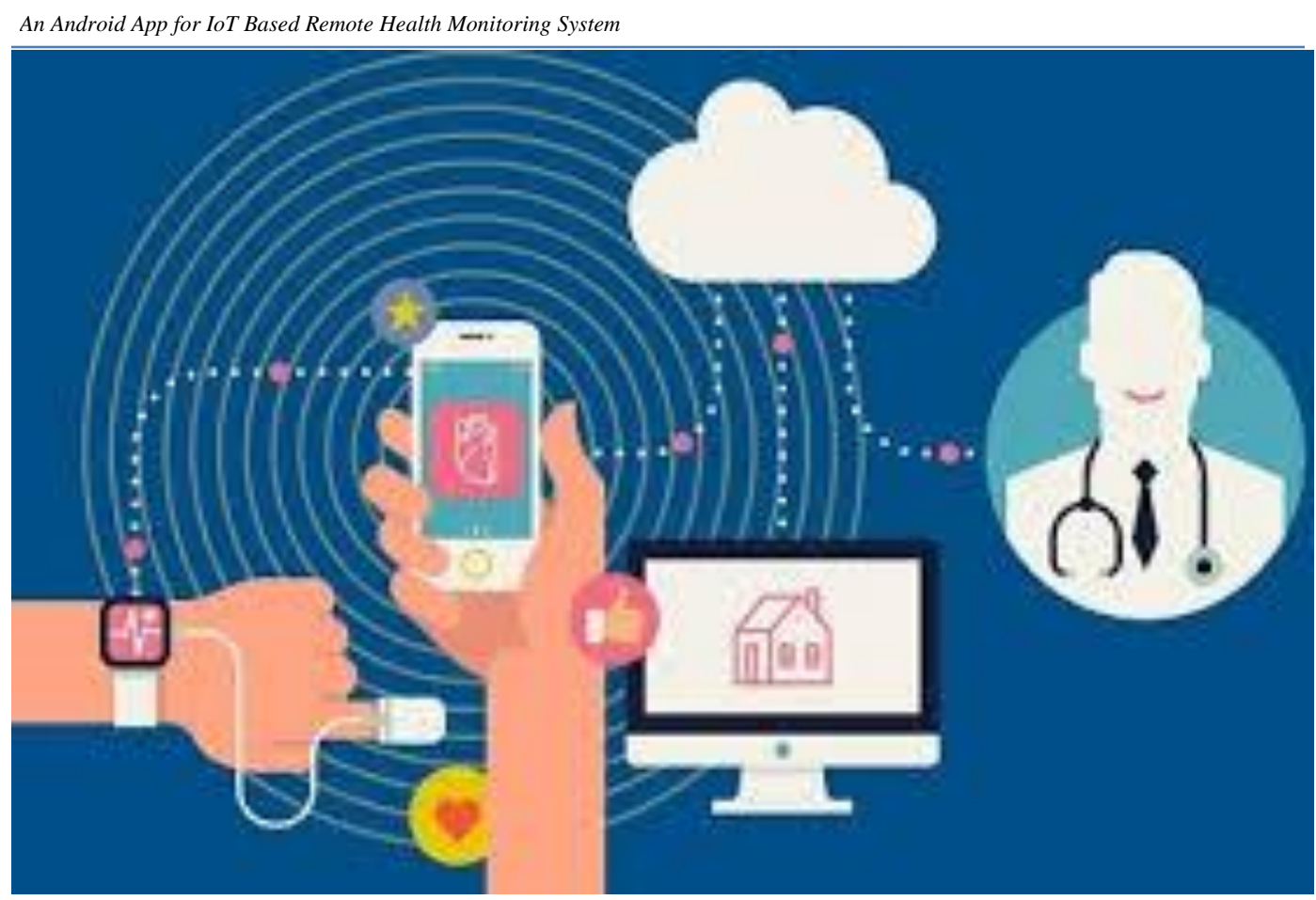

Figure-2: IoT in Health Sector Sample.

\section{Literature Survey}

With the development of the various technologies it is predicted that the availability of the remote monitoring system of internet is everywhere. These sensors have also less power consuming and able to perform the work like big health measurement machineries. Remote health monitoring conditions for patients have been carried out. Using Io $\mathrm{T}$ for this application is gaining a lot of scope off late. Io'T usage is what our focus is on. Priyantha et al [11] have recently showed the feasibility of SOAP-based Web services. Their work revealed several important insights into the interactions between Web services, the underlying TCP protocol, and power-saving MAC and link layer protocols. Our work has three major differences. First, we show that RESTful Web services, a much simpler mechanism than SOAP-based Web services, provide benefits in terms of completion time and power consumption. Second, we integrate theWeb services mechanism with an off-the-shelf power-saving MAC protocol (X$\mathrm{MAC}$ ) and provide important insights into optimizing its use for RESTful Web services. Third, we provide experimental results from a multi-hop network.

\section{OBJECTIVE}

the main objectives of this paper are as follows:

- Internet of Things (IoT) is the emerging technology, which contains huge amount of smart object and smart devices connected to the internet for communicating with each other. 
- In this project to analyze and compute the patient health we are using Raspberry Pi, which is the heart of this project.

- These smart devices are used to collect temperature and heartbeat which are used to evaluate the health condition of the patient.

- The final results are displayed on the android device, on web server and also the results are sent to the user through SMS.

- These data results can be stored in data base centre which can be invoked from remote location at any time in an emergency case of patient without delaying the time.

- This project may play vital role in saving the patient life at emergency time since "Time is life"

\section{System Requirements}

\section{HARDWARE REQUIREMENT}

- Raspberry pi 3

- Arduino

- DS18B20 Water proof digital temperature sensor

- INVNT_11 pulse rate sensor

- Bread board

- Jumper wires

\section{SOFTWARE REQUIREMENT}

- Rasbian OS

- $\quad$ Python 3 IDLE

- $\quad$ Arduino IDE

- $\quad$ Firebase cloud

- $\quad$ Sketchware software

\section{Proposed Methodology}

The temperature and pulse rate sensors are used to collect the external data. The collected data is organized and displayed using raspberry pi. For the analysis the data is stored in the cloud. The data is then sent to a sink which forwards the data to the required device like a smart phone, laptop. The stored data can be accessed by anybody using the ip address or the app. If any fluctuations are noted in the parameters, it will be alerted to the doctors and relatives through a notification.

Proceedings of the $3^{\text {rd }}$ National Conference on Image Processing, Computing, Communication, Networking and Data Analytics (NCICCNDA 2018) 


\section{Results}
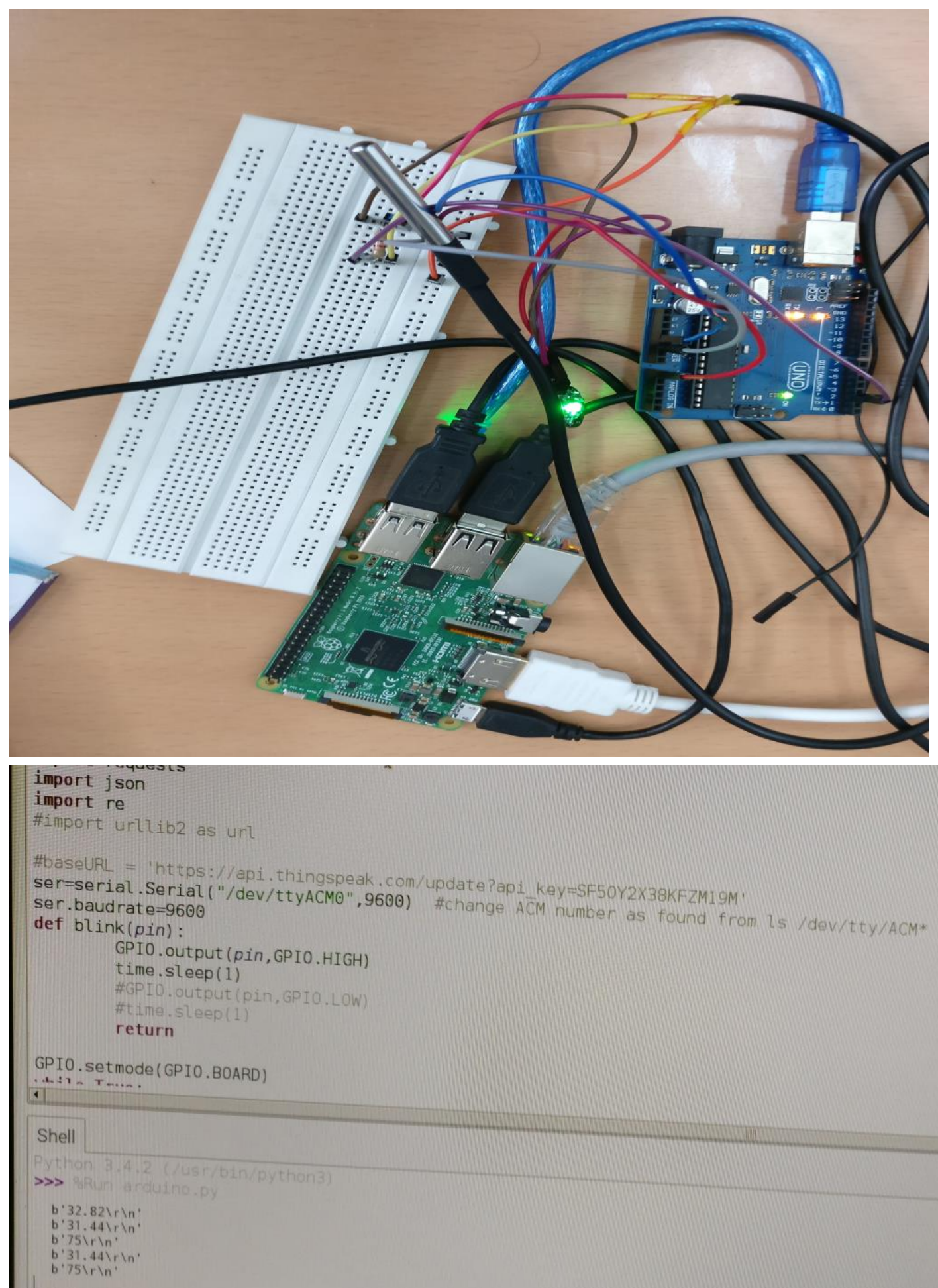

Figure-3: Proposed Design 
Smruthi et al, NCICCNDA 2018, AIJR Proceedings 1, pp.401-406, 2018

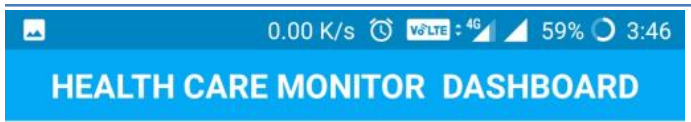

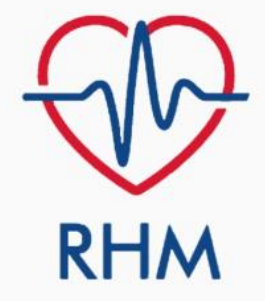

NAME

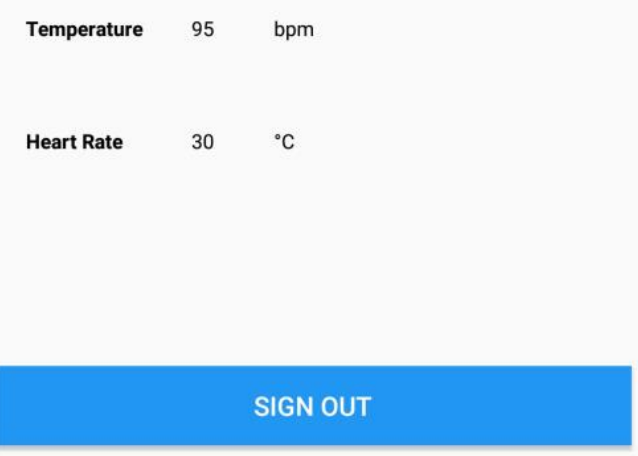

$\Delta$

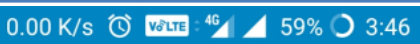

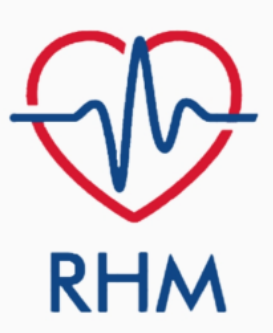

SIGN IN

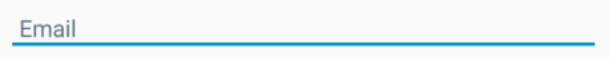

Password

LOGIN

SIGN UP

Enter password

Figure-4: APP of remote accessing

\section{7 conclusion}

This proposed work aims to give a in depth analysis of the IoT and its impact in health sector. As health care services are important part of our society, automating these services lessen the burden on humans and eases the measuring process. The objectives of this paper are to come out with a mobile app for remote health monitoring using the Arduino by reducing physician office visits, hospitalizations, and diagnostic testing procedure. Many further improvements can be made in our system to make it better and easily adaptable such as adding more advanced sensors. The future health sector of the humans can be efficiently influenced by the remote monitoring app and accessing applications.

\section{REFERENCES}

[1]J. Craig, V. Patterson, "Introduction to the practice of telemedicine," J.Telemed. Telecare, vol. 11, 1, pp. 3-9, 2005.

[2] Image, Signal, and Distributed Data Processing for Networked eHealth Applications Maglogiannis. I, Wallace. M,Karpouzis. K, Engineering in Medicine and Biology Magazine, IEEE, Vol. 26, Issue 5, Sept.-Oct. 2007 Page(s):14 $-17$

Proceedings of the $3^{\text {rd }}$ National Conference on Image Processing, Computing, Communication, Networking and Data Analytics (NCICCNDA 2018) 
An Android App for IoT Based Remote Health Monitoring System

[3] http://www.eecs.harvard.edu/ mdw/proj/codeblue/

[4] P. K. D. Marculescu, R. Marculescu. Challenges and opportunities in electronic textiles modeling and optimization. In Design Automation Conference, 2002. Proceedings. 39th,pages 175-180. ACM/IEEE, 2002

[5] J. E. T. Martin, M. Jones and R. Shenoy, "Towards a design framework for wearable electronic textiles. Seventh IEEE International Symposium on Wearable Computers,pages 190-199. IEEE, 2003

[6] Lifeguard monitoring system. http://lifeguard.stanford.edu

[7] R. Jafari et al., "Wireless Sensor Networks for Health Monitoring", The 2nd ACM/IEEE International Conference on Mobile and Ubiquitous Systems (MobiQuitous), July 2005,San Diego. CA

[8] A. Milenkoviü, C. Otto, E. Jovanov, "Wireless Sensor Networks for Personal Health Monitoring: Issues and an Implementation, " Computer Communications, Vol. 29, No. 1314, 2006, pp. 2521-2533

[9] G. Virone et al., "An Advanced Wireless Sensor Network for Health Monitoring," Transdisciplinary Conference on Distributed Diagnosis and Home Healthcare (D2H2),Arlington, VA, April 2-4, 2006

[10] X. Liang, I. Balasingham, "Performance Analysis of The IEEE 802.15.4 Based ECG Monitoring Network", IASTED International Conferences, Montreal, Quebec, Canada, 2007

[11] D. Bertsekas, R. Gallager, "Data Networks", Second Edition,Prentice Hall, 2001

[12] Banchs. A et al., "Applications and challenges of the 802.11e EDCA mechanism: an experimental study", Network, IEEE,Volume 19, Issue 4, July-Aug. 2005 Page(s):52 - 58

[13] Wookwon Lee et al., "On use of traditional M/G/1 model for IEEE 802.11 DCF in unsaturated traffic conditions", IEE WCNC 2006, Volume 4, Page(s):1933 - 1937

[14] Zhifeng Tao and Panwar. S, "Throughput and delay analysis for the IEEE 802.11e enhanced distributed channel access,'IEEE Transactions on Communications, April 2006 Volume54, Issue 4, on page(s) 596- 603 